\title{
Vacancy in Silicon Revisited: Structure and Pressure Effects
}

\section{Citation}

Antonelli, A., Efthimios Kaxiras, and D. Chadi. 1998. “Vacancy in Silicon Revisited: Structure and Pressure Effects." Physical Review Letters 81 (10): 2088-91. https://doi.org/10.1103/ physrevlett.81.2088.

\section{Permanent link}

http://nrs.harvard.edu/urn-3:HUL.InstRepos:41384110

\section{Terms of Use}

This article was downloaded from Harvard University's DASH repository, and is made available under the terms and conditions applicable to Other Posted Material, as set forth at http:// nrs.harvard.edu/urn-3:HUL.InstRepos:dash.current.terms-of-use\#LAA

\section{Share Your Story}

The Harvard community has made this article openly available.

Please share how this access benefits you. Submit a story.

Accessibility 


\title{
Vacancy in Silicon Revisited: Structure and Pressure Effects
}

\author{
A. Antonelli \\ Instituto de Física Gleb Wataghin, Universidade Estadual de Campinas, Unicamp, 13083-970 Campinas, São Paulo, Brazil \\ Efthimios Kaxiras \\ Department of Physics and Division of Engineering and Applied Sciences, Harvard University, Cambridge, Massachusetts 02138 \\ D. J. Chadi \\ NEC Research Institute, 4 Independence Way, Princeton, New Jersey 08540-6634
}

(Received 27 February 1998; revised manuscript received 15 June 1998)

\begin{abstract}
The structure of the single vacancy in silicon, one of the most common point defects and an important mediator of atomic diffusion, is examined through extensive first principles calculations. We find a hitherto unexpected result, namely that there exist two distinct distortions associated with the vacancy with essentially identical formation energies at zero pressure. The two distortions are distinguished by their different relaxations, volumes of formation, and formation enthalpies. We discuss how, at finite pressure, one of the two distortions should become dominant, and suggest experimental tests of this effect. [S0031-9007(98)07002-1]

PACS numbers: 61.72.Ji, 66.30.Hs, 66.30.Jt
\end{abstract}

The study of native point defects in semiconductors has attracted a great deal of attention in the last 20 years. In part this was due to the role played by such defects in mediating atomic diffusion, a process of fundamental importance in the doping of semiconductors. Despite their apparent simplicity, the determination of basic structural and electronic properties of these defects has turned out to be a challenging problem.

A case in point is the single vacancy in $\mathrm{Si}$ which has been the subject of extensive theoretical and experimental studies. The importance of relaxations of the crystalline lattice around the Si vacancy and its negative- $U$ nature were pointed out theoretically [1] and confirmed experimentally [2] almost two decades ago. More efficient and accurate computations have provided a better picture of the structure of the single neutral vacancy. The initial suggestion of an outwards relaxation of the neighbors (i.e., away from the vacant site) [1], has been superseded by detailed $a b$ initio total energy calculations [3], that show an inward relaxation (i.e., toward the vacant site). Subsequent studies [4] have determined that this inward breathing relaxation around the vacancy is larger than previously estimated. Results from semiempirical calculations at zero temperature [5] indicate a Jahn-Teller tetragonal distortion $\left(D_{2 d}\right)$, while simulated annealing at finite temperature [6] gives a distortion corresponding to a $T_{2}$ mode of the trigonal distortion, a result predicted theoretically [7], but not observed experimentally. More accurate first-principles calculations [8,9] have confirmed the Jahn-Teller tetragonal distortion. Very recently, thorough first-principles calculations [10] have shown that trigonal distortions are unstable and that tetragonal and tetrahedral distortions can occur. Despite the progress, some aspects of this complicated picture still have to be unraveled, such as the relative stability and dominance of the various distortions.

In this Letter, we report a thorough investigation of the relaxation of the Si neutral vacancy and its formation volume, using first-principles total energy calculations. Our work reveals yet another unexpected result: There are actually two distinct distortions induced by the vacancy, which have practically identical formation energies at zero pressure, but substantially different volumes of formation and therefore different formation enthalpies. Consequently, at finite pressure one of the two distortions becomes dominant. We propose that since the two distortions yield formation volumes with opposite signs it should be possible to observe them experimentally through nonhydrostatic stress measurements.

The calculations were performed within local density functional theory with nonlocal pseudopotentials and a plane wave basis. The two distortions were initially obtained using a basis including plane waves with kinetic energy up to $16 \mathrm{Ry}$. The analysis of volume and pressure effects was carried out with a basis including plane waves with kinetic energy up to $10 \mathrm{Ry}$, since we have found that the magnitude and character of the distortions does not change significantly with the smaller basis. Periodic supercells with 64 and 216 atoms were employed [with a $2 \times 2 \times 2$ uniform grid of $k$ points, and the $(1 / 4,1 / 4,1 / 4)$ special point in the Brillouin zone, respectively]. The larger unit cell was used selectively to ensure that finite size effects were not important. Atomic relaxations were considered to the point where the magnitude of the calculated Hellmann-Feynman forces on each atom was smaller than $0.002 \mathrm{Ry} / \mathrm{a}$.u. The formation volume was obtained by calculating the energy as a function of the lattice parameter of a crystal containing 
one vacancy per unit cell, including full atomic relaxation for lattice parameters close to the equilibrium one.

There are four broken (dangling) bonds around a single vacancy in $\mathrm{Si}$, which gives rise to a nondegenerate $A_{1}$ level and a triply degenerate $T_{2}$ level. The $A_{1}$ level contains two electrons and is resonant with the valence band, while $T_{2}$, also containing two electrons, lies in the band gap. The tetragonal Jahn-Teller distortion is shown in Fig. 1. The four neighbors of the vacancy, labeled 1, 2, 3, and 4 , move away from the sites they occupy in the perfect crystal forming two pairs (atom 1 with 2, and atom 3 with 4), as indicated by the arrows shown in Fig. 1. This distortion breaks the tetrahedral symmetry of the system and the degeneracy of the $T_{2}$ level, lowering the energy of the occupied orbital, and raising the energy of the other two unoccupied orbitals. This distortion can be seen as a rebonding of the atoms in order to eliminate the dangling bonds. The rebonding effect is illustrated in Fig. 2, where the valence charge density indicates the formation of bonds between the two atoms in each pair, at the expense of weakening their back bonds, which are significantly stretched. The pairs of atoms can move relative to one another, and are usually considered to move apart, justified by the fact that in each pair the atoms have been rebonded. Although insightful and interesting, this analysis based on group theory and chemical bonding cannot capture the full complexity of the system, which also includes the strong interaction of the vacancy and its neighbors with the rest of the crystal. The latter interaction leads to the two different distortions revealed by our calculations.

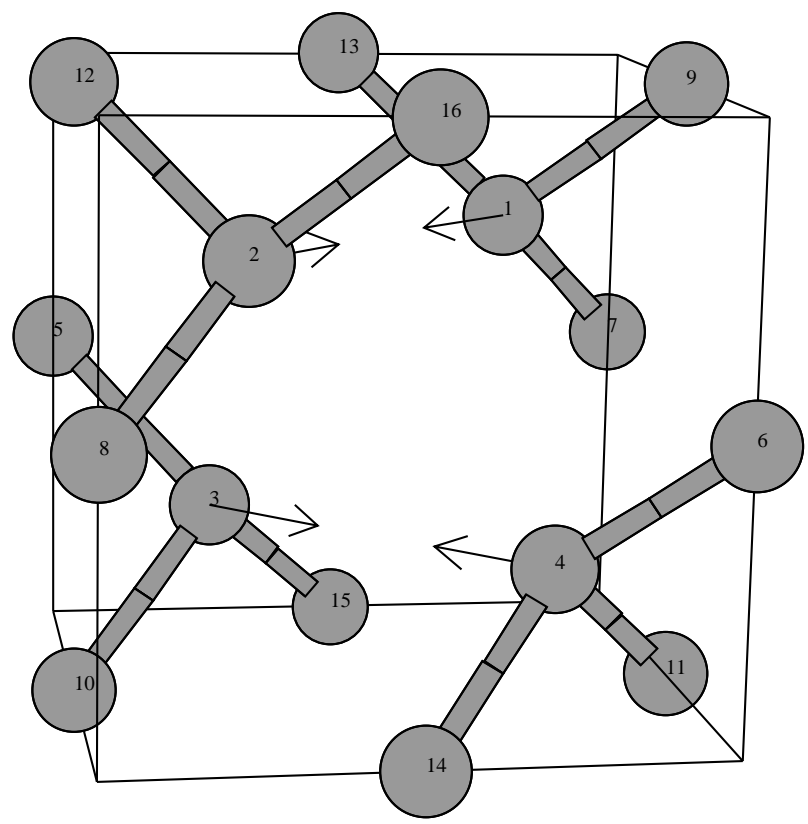

FIG. 1. Crystalline structure of the unrelaxed vacancy in $\mathrm{Si}$, showing the atoms that are first and second nearest neighbors of the vacancy. The arrows indicate the pairing of first nearest neighbors.
We discuss first the features of the two distortions at fixed volume ( $20 \AA^{3}$, the experimental equilibrium volume per atom). In the first distortion, labeled $A$, the distance between the atoms in each pair is $3.01 \AA$ and the distance between atoms belonging to different pairs is $3.53 \AA$. In this case, the two pairs of atoms move away from one another. In the second distortion, labeled $B$, although the original tetrahedral symmetry is also broken, that occurs to a much lesser extent than in the case of distortion $A$, with the distance between atoms in a pair at $3.40 \AA$, and the distance between atoms in different pairs at $3.52 \AA$. In this case the two pairs of atoms move toward one another. The bond between the atoms in each pair is significantly weaker in distortion $B$ than in distortion $A$, as shown in Fig. 2. The energy of distortion $B$ is lower than that of distortion $A$ by $0.1 \mathrm{eV}$. The Hellman-Feynman forces in both cases are below $0.002 \mathrm{Ry} / \mathrm{au}$, indicating that the two distortions correspond to equilibrium configurations. This result was checked using the 216-atom cell. We have investigated the possibility that the two distortions correspond to a stable state and a metastable state separated by an energy barrier, by interpolating between the distortions through a continuous path and calculating the energy along this path. We find no energy barrier along this path at the chosen fixed volume. Therefore, for this volume, distortion $A$ is an unstable equilibrium configuration. We are able to stabilize the system at distortion $A$ because we are performing zero temperature calculations. Otherwise, the system would evolve to distortion $B$ lowering its energy.

Since the two distortions around the vacancy are substantially different, the volume relaxation of the crystal can be different in each case. Our energy-versus-volume calculations indicate that indeed the two distortions have different equilibrium volumes, although the bulk modulus, its derivative, and the minimum energy are the same within the accuracy of our calculations. The volume relaxation with respect to the perfect crystal for distortion $A$ is $-21.4 \AA^{3}$, where the negative sign indicates a contraction of the system upon the formation of the vacancy in this configuration. The volume relaxation for distortion $B$ is substantially smaller in magnitude, $-15.5 \AA^{3}$. The atomic volume for the perfect crystal, determined from our calculations, is $19.7 \AA^{3}$ which compares very well with the experimental value of $20 \AA^{3}$. Adding the atomic volume of the perfect crystal to the volume relaxation of the crystal with the vacancy (which in the thermodynamic limit corresponds to placing the atom taken from the vacant site to a surface site) gives the vacancy formation volume, which is $-1.7 \AA^{3}$ for distortion $A$ and $+4.0 \AA^{3}$ for distortion $B$.

Since the total energy of the perfect crystal and that with vacancies have essentially the same dependence on volume, the internal formation energy of the vacancy does not depend on the volume of the system (for either distortion). But because the two distortions have different formation volumes, the formation enthalpy of the vacancy 
(a)

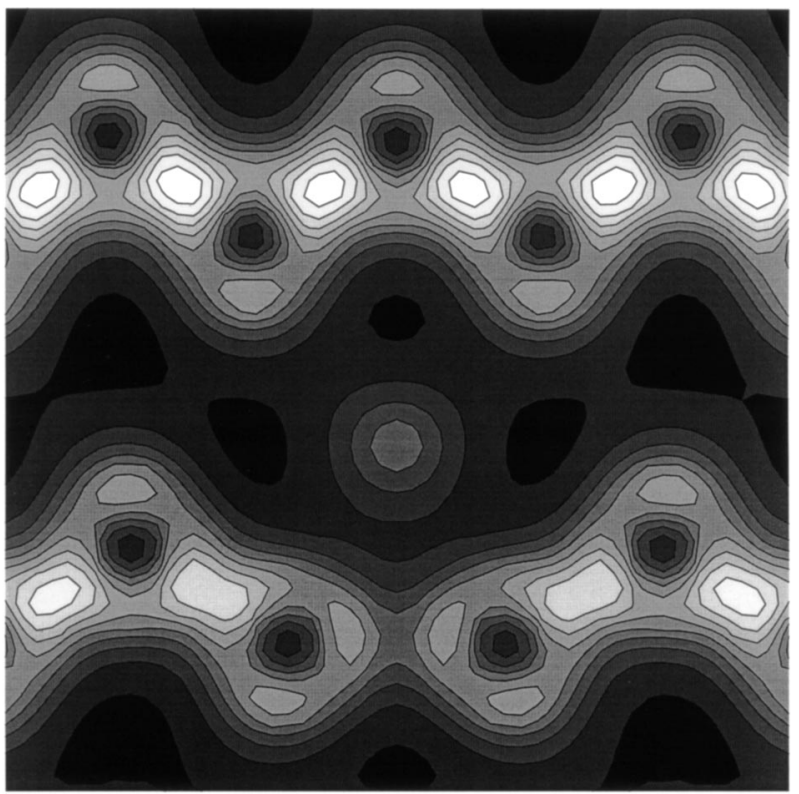

(b)

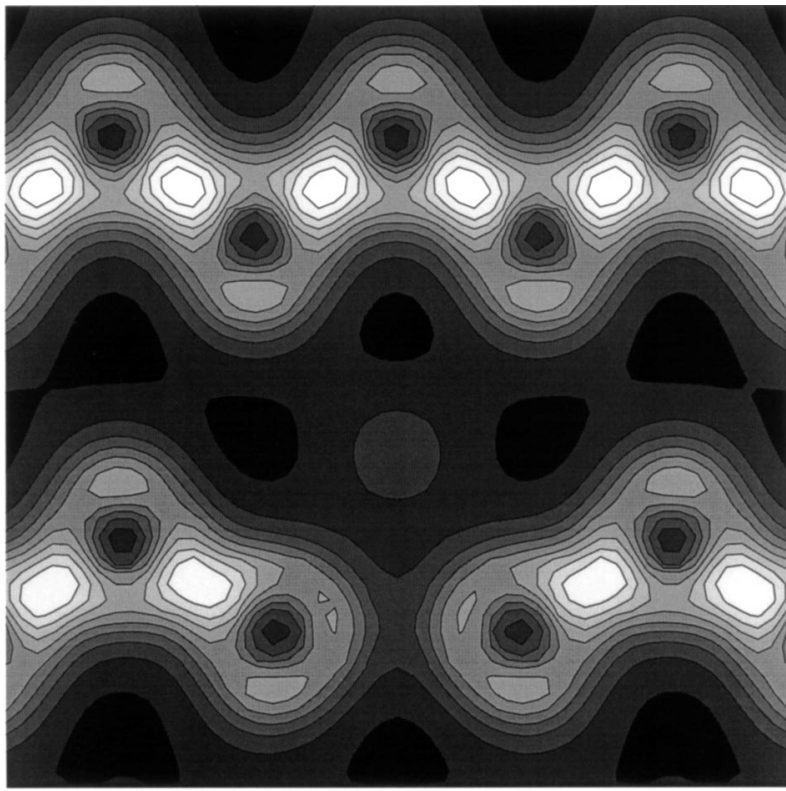

FIG. 2. Valence charge density on a (110) plane of the crystal which passes through the vacancy site at the midpoint of the lower chain of atoms. The chain of atoms in the upper half serves as a reference for atomic positions and bonds in a nearly ideal configuration. (a) Distortion $A$ : the nearest neighbors of the vacant site approach each other and form bonds along the chain, stretching their back bonds. The circular charge distribution near the center of the figure is a cross section of the bond between the two nearest neighbors of the vacancy which lie on either side (in front and behind) of the plane of the figure. (b) Distortion $B$ : the bond between the atoms in each pair is much weaker for this distortion, and the back bonds of these atoms are less stretched.

as a function of pressure is different for each distortion. In Fig. 3 we show the calculated formation enthalpy of the vacancy as a function of pressure. Since distortion $A$ has a negative formation volume the enthalpy of

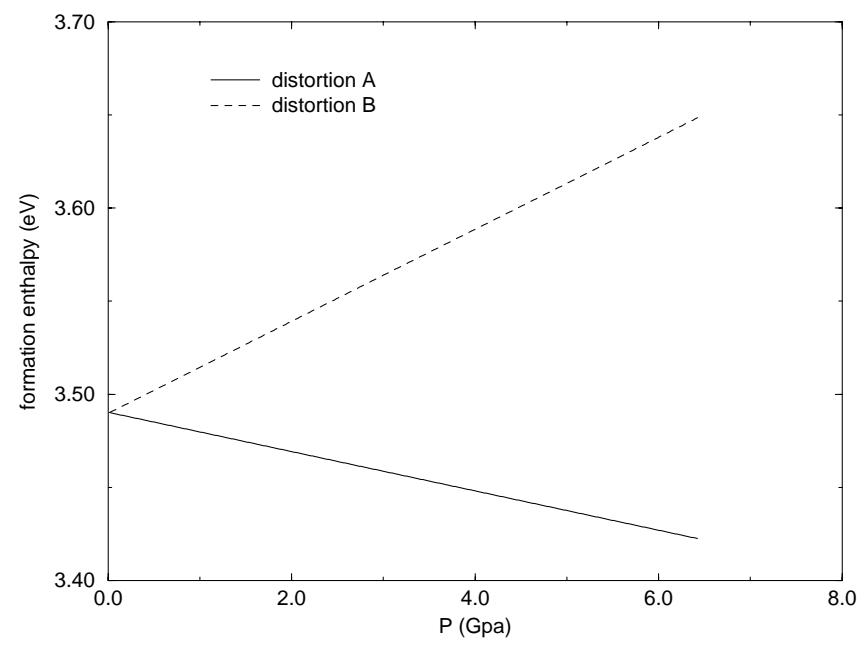

FIG. 3. Formation enthalpy as a function of pressure for distortion $A$ (solid line) and distortion $B$ (dashed line).

formation in this case decreases with pressure, whereas the formation enthalpy for distortion $B$ increases with pressure, due to its positive formation volume. Two interesting conclusions can be drawn from these results. First, the two distortions can coexist at zero pressure, since the formation enthalpy at zero pressure is the same. There is, in fact, a small energy barrier separating the two distortions: the energy-versus-volume curves of the two distortions are offset and intersect, the point of intersection being this barrier as a function of volume, with the two minima on either side of it. This barrier, which according to our calculations is of the order of a few $\mathrm{meV}$, can be easily overcome by thermal excitation. Second, as pressure is applied, distortion $A$ will dominate, since its formation enthalpy decreases with pressure, whereas the formation enthalpy of distortion $B$ increases. At a pressure of $5 \mathrm{GPa}$, for instance, the difference in formation enthalpy for the two distortions is of the order of $0.2 \mathrm{eV}$; therefore, in this pressure range and even at high temperatures, distortion $A$ should be dominant. In these estimates we are not taking into consideration entropic effects which are difficult to estimate from static calculations; these effects can be relevant at high temperatures.

To make contact with experiment we consider the effect of vacancies in dopant diffusion, which is an indirect but widely used approach to infer the properties of point defects. The measured activation volume for diffusion of As in Si [11] is $\approx-9 \pm 4 \AA^{3}$. The activation energy for the vacancy mechanism includes the formation energy of an isolated vacancy, the migration energy for the vacancyimpurity complex, and the dissociation energy of this complex [12]. By analogy, the activation volume for As diffusion in $\mathrm{Si}$ includes the formation volume of an isolated vacancy and the contributions from migration and dissociation of the vacancy-impurity complex. The 
experimentally measured large and negative activation volume was a puzzling result, since it was thought that the formation volume of a vacancy should be positive, with a magnitude close to one atomic volume (i.e., $\sim 20 \AA^{3}$ ). An early total energy ab initio calculation found a positive formation volume of the vacancy in $\mathrm{Si}$ of $+15 \AA^{3}$ [3]. On this basis, it was argued that if As diffusion in $\mathrm{Si}$ were mediated by vacancies, the sum of the migration and dissociation volumes should be quite large and negative. More recent calculations started to change this picture. Sugino and Oshiyama [4] obtained a large inward relaxation of the nearest neighbors of the vacancy, which indicates that the formation volume of the vacancy could be negative, with an estimated magnitude of $-5 \AA^{3}$. This result is obtained by considering the total change in the volume of the crystal to be equal to the local change in volume around the vacancy. This conclusion, however, requires the assumption that the crystal is a continuous elastic medium. Very recent calculations using constant pressure tight-binding molecular dynamics simulations found a small positive formation volume of $+0.6 \AA^{3}$ [13]. In our calculations, distortion $A$ will dominate as pressure is applied, therefore our estimate for the measured vacancy formation volume is $-1.7 \AA^{3}$. Our first-principles calculations confirm that the volume relaxation upon vacancy formation is indeed quite large, in qualitative agreement with previous estimates $[4,13]$. It should be emphasized that our results do not rely on approximating the crystal as a continuous elastic medium. These theoretical results indicate that no large and negative contribution from migration and dissociation to the activation volume needs to be assumed. The negative activation volume for As diffusion in $\mathrm{Si}$ is indeed consistent with a dominant vacancy mechanism.

Experimental observation of the two distortions predicted by our calculations can be attempted through diffusion measurements in thin films under biaxial stress. Experiments of this type have been reported for diffusion of Sb in strained $\mathrm{Si}$ and $\mathrm{SiGe}$ [14]. Following the analysis of Aziz [15], the change in the equilibrium concentration of vacancies in biaxially strained films is given by

$$
C^{e}(\sigma)=C^{e}(0) \exp \left(2 \sigma V^{r} / 3 k T\right),
$$

where $\sigma$ is the biaxial stress and $V^{r}$ the relaxation volume of the system upon the formation of the defect. In this case, since the surface is considered free, placing an atom on it does not require the work needed in the hydrostatic case. This analysis provides a way to relate the relaxation volume obtained from experiments or calculations in hydrostatic conditions with the vacancy concentration in a system under biaxial stress. However, the analysis is based on the assumption that the crystal can be considered a continuous elastic medium and neglects the microscopic details of the crystal. Although limited, we believe this analysis can provide some useful insight on the experimental observation of the two distortions. Since the relaxation volume of distortion $A$ is around $30 \%$ larger than that of distortion $B$, the observed reduction of the diffusion under tensile strain would be smaller than the increase under compressive strain, if we assume that migration parameters are unchanged by the sign of the biaxial stress. This effect was not seen in the experiments reported so far [14], but we suggest that this is a consequence of the small differences (as predicted by our calculations) which are smaller than experimental error bars. We expect that more accurate experiments should be able to detect this effect.

We thank Professor M. J. Aziz for useful discussions throughout this work and helpful comments on the manuscript. A. A. wishes to thank Harvard University for its hospitality during the course of this work, and to acknowledge partial support from the Brazilian funding agencies Fundo de Apoio ao Ensino e à Pesquisa (FAEP/UNICAMP), Fundação de Amparo à Pesquisa do Estado de São Paulo (FAPESP), and Conselho Nacional de Desenvolvimento Científico e Tecnológico (CNPq). Computer calculations were performed at the facilities of the Centro Nacional de Processamento de Alto Desempenho em São Paulo (CENAPAD-SP). The work at Harvard University was supported the Materials Science and Engineering Center, which is funded by the National Science Foundation.

[1] G. A. Baraff, E. O. Kane, and M. Schlüter, Phys. Rev. B 21, 5662 (1980).

[2] G.D. Watkins and J.R. Troxell, Phys. Rev. Lett. 44, 593 (1980).

[3] A. Antonelli and J. Bernholc, Phys. Rev B 40, 10643 (1989).

[4] O. Sugino and A. Oshiyama, Phys. Rev. B 46, 12335 (1992).

[5] C.Z. Wang, C. T. Chan, and K. M. Ho, Phys. Rev. Lett. 66, 189 (1991).

[6] E. G. Song, E. Kim, and Y.H. Lee, Phys. Rev. B 48, 1486 (1993).

[7] J. Bourgoin and M. Lannoo, Point Defects in Semiconductors II (Springer, Berlin, 1983), Vol. 35.

[8] H. Seong and L. Lewis, Phys. Rev. B 53, 9791 (1996).

[9] S. Öğüt, H. Kim, and J. R. Chelikowsky, Phys. Rev. B 56, R11 353 (1997).

[10] L. J. Mercer, J. S. Nelson, A. F. Wright, and E. B. Stechel, Model. Simul. Mater. Sci. Eng. 6, 1 (1998).

[11] E. Nygren, M. J. Aziz, D. Tunbull, J.M. Poate, D.C. Jacobson, and R. Hull, Appl. Phys. Lett. 47, 105 (1985).

[12] S. M. Hu, Phys. Status Solidi (b) 60, 595 (1973).

[13] M. Tang, L. Colombo, J. Zhu, and T. D. de la Rubia, Phys. Rev. B 55, 14279 (1997).

[14] P. Kringhøj, A. N. Larsen, and S. Y. Shirayev, Phys. Rev. Lett. 76, 3372 (1996).

[15] M. J. Aziz, Appl. Phys. Lett. 70, 2810 (1997). 